Precision Engineering, Volume 60, November 2019, pp. 368-373

DOI:10.1016/j.precisioneng.2019.09.002

\title{
Improved and simpler estimation of scale linearity contribution to topography measurement
}

\author{
Claudiu L Giusca* and Saurav Goel \\ Surface Engineering and Precision Institute, Cranfield University, Cranfield, \\ Bedfordshire MK430AL, UK \\ *Corresponding author: c.giusca@cranfield.ac.uk
}

\begin{abstract}
Instruments measuring surface topography with nanometre accuracy are essential tools for studying nanotechnology. Despite their maturity, erroneous observations due to various error sources are widespread, particularly due to calibration and traceability issues. The current method of vertical scale calibration (which is one of the error sources), relies on the depth standard method that limits the traceability of the instrument to the calibrated range determined by the minimum and maximum discrete values. This paper reports a new method relying on the tilted flat that was aimed at mapping the linearity deviations continuously within the range covered by the tilt angle. The full traceability in this case requires only the measurement of a single depth measuring standard, that can be optimally selected to achieve least uncertainty associated with the amplification coefficient of the scale. The proposed method opens the opportunity for high dynamic range calibration, currently unachievable with conventional calibration techniques.
\end{abstract}

\section{Introduction}

Surface topography measurement has become an important technique for a wide range of fields such as nano- and micro- fabrication, biomedical sciences, optics, general material characterisation, just to list a few $[1,2]$. Owing to the ability of most common surface topography instruments, such as Atomic Force Microscopes (AFM), Coherence Scanning Interferometers (CSI) or optical microscopes based on confocal principles, of providing sub-nanometre vertical resolution [3], the technique has also been seen to enable new and disruptive technologies such as quantum computing [4]. Although these state-of-the-art instruments such as the AFM and optical microscopes can discriminate between the tiniest surface asperities, the vertical resolution alone is not the only major error source affecting topography assessment [5] and in selective cases, vertical scale calibration is seen to be an issue.

Most of the surface topography instruments rely on vertical scanning i.e. displacing and recording the position of the probe, such as the cantilever tip or an objective lens, relative to the measured surface in the vertical direction. Vertical scale calibration evaluates the effect of the errors associated with linearity and the amplification coefficient of the scale. Sometimes, features on the measured sample provide means of 
estimating such errors, for example presence of atomic steps, however in most cases proper calibration of an instrument, in particular of its scale is so extremely necessary because otherwise a series of irreversible manufacturing and measurement errors and deceptive conclusions are possible. The subject in itself has a long lineage and numerous attempts have been made to resolve errors arising during the calibration stage but only limited success is evident to date. Taking the example of a linear vertical scale measurement, this paper proposes a novel and simplified procedure for determining the linearity of the vertical scale, hitherto unaddressed in the extant literature. This new finding will aid the continuous calibration of the vertical scale with the added benefit of providing complete information necessary for modern process chains, as measurement uncertainty instead of simple standard deviations, which do not accommodate for biases.

The rich literature in the field details a wide range of absolute and relative measurement techniques able to estimate the effect of different error sources using interferometers, error separation methods or calibrated artefacts $[6,7,8]$. Some artefacts already include an inclined surface [9,10], which is required for the method presented in this paper. However, when it comes down to propagating the effect of these error sources to the measurement uncertainty with due diligence, the proposed calibration process lacks pragmatism. For example, the vertical scale of the surface topography measuring instruments is often achieved using a limited number of calibrated depth standards (Type PGR standards according to ISO 25178 [11]) [12] or using interferometers [6]. However, these methods are either expensive, impractical, do not provide sufficient information or a combination of the above. More recently, the demand for accurate topographical measurements of optical components lead researchers [13] to use a tilted surface to calculate the measurement uncertainty caused by surface gradient.

Competition in the field has grown stiffer and instrument manufacturers seem more concerned about assuring the linearity of the vertical scales, and with the luxury of a calibrated laser source they are able to provide continuous calibration of the vertical scales that is not permitted by simple depth measurement standards [14]. Most notably, Kiyono [15] proposed a method that allows correcting long wavelength nonlinearities present in the vertical scales using a translated tilted plane, however, the method requires the use of a displacement interferometer and is restricted by the size of the shift, which limits sampling frequency at which the vertical scale can be calibrated. Based on an error separation technique, nonlinearities in the vertical scale can be depicted with an inclined flat $[16,17]$, however, at the microscale rotational and reposition accuracy is difficult to achieve.

In this paper, it was hypothesised that a near flat inclined surface can depict the linearity of the vertical scale very accurately, with the caveat that the effects of other influencing parameters which tend to distort the measured topography [18] may not be fully isolated. However, such rich information provides the ability to construct seamless uncertainty budgets fitted to serve modern technology endeavours with affordable economic costs for the instrument manufacturer and end users. 


\section{Theory}

The measurement of a tilted plane can simply be modelled in two dimensions by the following equations:

$$
\begin{aligned}
& { }^{{ }^{i n d}} z=\alpha_{z}{ }^{T} z+l_{z}\left({ }^{T} z\right)+z_{\mathrm{FLT}}+N_{\mathrm{M}}+z_{\text {sample }}, \\
& { }^{T} Z=\left(\alpha_{x}{ }^{T} x+l_{x}\left({ }^{T} x\right)\right) \tan \theta
\end{aligned}
$$

Where (see figure 1):

${ }^{\text {ind }} Z$ - measured profile (2D topography),

$\left({ }^{T} Z,{ }^{T} X\right)$ - input quantities tested within the measurement range of the vertical and lateral scales,

$\alpha_{z}$ and $\alpha_{x}$ - corresponding amplification coefficients,

$l_{z}\left({ }^{T} Z\right)$ and $l_{X}\left({ }^{T} X\right)$ - linearity deviation of the scales,

$\theta$ - tilt angle of the plane (sample),

$Z$ FLT - flatness deviation of the instrument,

$Z$ sample - intrinsic topography of the sample,

$N_{\mathrm{M}}$ - measurement noise.

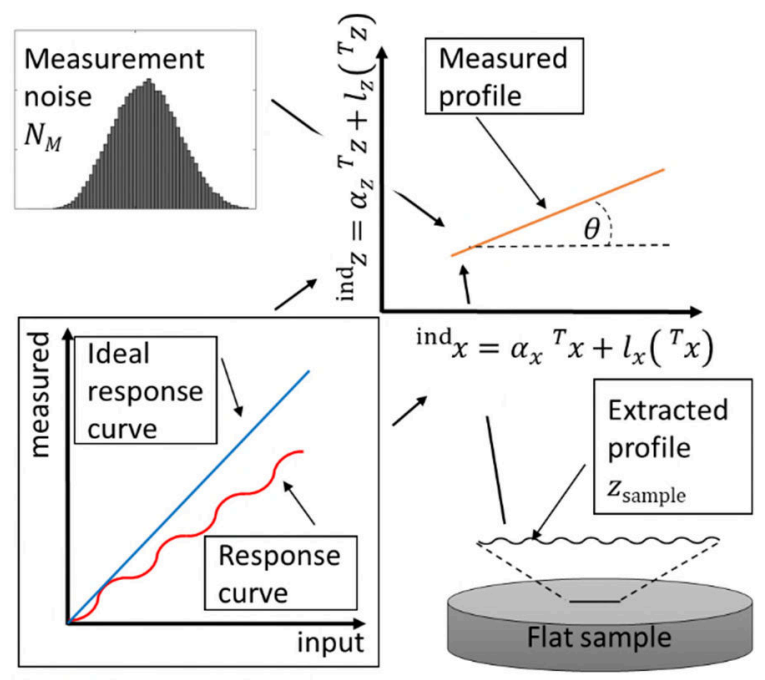

Figure 1 Graphical representation of the input parameters accounted in equations 1 and 2.

Levelling the measurement result, which mathematically is equivalent to

$$
{ }^{\text {ind }-0} z={ }^{\text {ind }} z-\alpha_{z} \alpha_{x}{ }^{T} x \tan \theta \text {, }
$$

eliminates the first order terms of the polynomial. Hence, equation 1 can be rewritten in the flowing form:

$$
{ }^{\text {ind }-0} z=l_{z}\left({ }^{T} z\right)+\alpha_{z} l_{x}\left({ }^{T} x\right) \tan \theta+z_{\mathrm{FLT}}+N_{\mathrm{M}}+z_{\text {sample }}
$$

Equation 4 shows that the levelled measured profile, ${ }^{\text {ind }}-0 z$, can be used to estimate the magnitude of the $z$ scale linearity deviation $\left(l_{z}\right)$, as long as the rest of the parameters are small compared to $l_{z}$.

Unlike the Kiyono's method [15], sample topography, flatness deviation and lateral scale linearity deviation will affect the estimation of the vertical scales linearity. However, the 
influence of these three parameters can be mitigated using a good quality optical flat and/or additional measurements, allowing minimisation of the effect of such systematic errors.

A more subtle benefit of equation 4 is that one can ascertain quantitatively the summary information for the PDF associated with $l_{z}$, namely means and standard deviations (variances). Using the law of propagation of uncertainties in measurement [19], one can derive the next equation:

$$
\begin{aligned}
u_{\mathrm{ind}-0}^{2}=u_{l_{z}}^{2} & +\left(\alpha_{z} \tan \theta\right)^{2} u_{l_{x}}^{2}+\left(l_{x} \tan \theta\right)^{2} u_{\alpha_{z}}^{2} \\
& +\left(\alpha_{z} l_{x} \sec ^{2} \theta\right)^{2} u_{\theta}^{2}+u_{z_{\mathrm{FLT}}}^{2}+u_{N_{\mathrm{M}}}^{2}+u_{z_{\text {sample }}}^{2}
\end{aligned}
$$

where, $u$ stands generally for standard uncertainty and in combination with the subscripts indicates the standard uncertainty associated with each of the input and output quantities specified in equation 4 . For example, $u_{N_{\mathrm{M}}}$ is the uncertainty associated with the effect of the measurement noise.

The measurement of the tilted flat is equivalent to measuring the linearity deviation of the $\mathrm{z}$ scale within a specific measurement volume and with a sampling interval limited by the sampling interval of the $x$ axis multiplied by the tangent of the tilt angle. For example, in the case of a tilted flat covering a $\mathrm{z}$ (vertical) range of $1 \mu \mathrm{m}$ that is evaluated over $1 \mathrm{~mm}$ in the $x$ (horizontal) direction with a sampling interval of $1 \mu \mathrm{m}$, the equivalent sampling of the linearity deviation of the $\mathrm{z}$ scale is $1 \mathrm{~nm}$. The measured profile will consist of a discreet set of points, $\left\{{ }^{\text {ind }-0} Z_{i}\right\}_{i=1: n}$, where $n$ is given by the horizontal length of the profile divided by the sampling interval. Consequently, from the set of differences $\left\{\left({ }^{\text {ind }-0} \mathbf{Z}_{i+j^{-}} \text {ind- } 0 Z_{i}\right)\right\}_{i=1: n-j, j<n}$ means and variance that summarises the PDF associated with the linearity deviation effect on the measurement height of $\left({ }^{\text {ind }-0} \mathrm{Z}_{i+j}\right.$ - ind${ }^{0} Z_{i}$ ), can be estimated. Calling on the previous example where $n$ is 1000 , if $j$ is equal to 10 , then one can calculate the mean and variance associated with the linearity deviation contribution to the measurement of a $10 \mathrm{~nm}$ height.

It is evident from equation 5 that the variance will also include the effect of the measurement noise, flatness deviation and so on, however, one can estimate the combined variance of most of these parameters by measuring the sample without tilt which is modelled by:

$$
\text { ind }_{z_{0}} \cong z_{\mathrm{FLT}}+N_{\mathrm{M}}+z_{\text {sample }}
$$

where ${ }^{\text {ind }} z_{0}$ is the measured profile without tilt.

The exact estimate of the variance associated $l_{z}$ can easily be calculated as the difference between the variance calculated from the measurement of a tilted flat, $\left\{\left({ }^{\text {ind }-0} \mathrm{Z}_{i+j}{ }^{\text {ind }}-0 Z_{i}\right)\right\}_{i}=$ $1: n-j, j<n$, and the variance from the measurement of the flat without tilt, $\left\{\left(\mathrm{Cind}_{\mathrm{Z} 0, i+j}{ }^{\text {- ind- }}\right.\right.$

$\left.\left.{ }^{0} Z 0, i\right)\right\}_{i=1: n-j, j<n}$.

The only contributions left unaccounted for in equation 4 are the ones corresponding to the uncertainty associated with $\alpha_{z}, l_{x}\left({ }^{\mathrm{T}} x\right)$ and $\theta$. However, at small tilt angles these terms have negligible effects. Nevertheless, one should crudely estimate the magnitudes of their means and variances to check if they have significant contributions. These pieces 
of calculations assemble as a connecting missing link for taking up the research problem of linear scale calibration formulated and described above. Consequently, the methods and results described in the next section are based accordingly.

\section{Methods}

A silicon carbide flat was measured using a contact stylus instrument Taylor Hobson Form Talysurf series 2 fitted with a $2 \mu \mathrm{m}$ radius conispherical tip. The vertical resolution of the instrument was limited by an $8 \mathrm{~nm}$ digitisation value and the horizontal sampling interval was set at $250 \mathrm{~nm}$ [20]. The measurements were analysed with a commercial surface topography analysis software, Mountains version 7 provided by Digital Surf.

\section{Results}

Figure 2 shows an example of the measurement of a silicon carbide flat without tilt (near flat sample) that was polished and provided by the instrument manufacturer as a measurement standard that allows calibrating the flatness deviation. Interestingly, it was observed that the $\mathrm{z}$ scale digitisation was larger compared to flatness deviation, measurement noise and amplitude of the intrinsic topography of the flat.

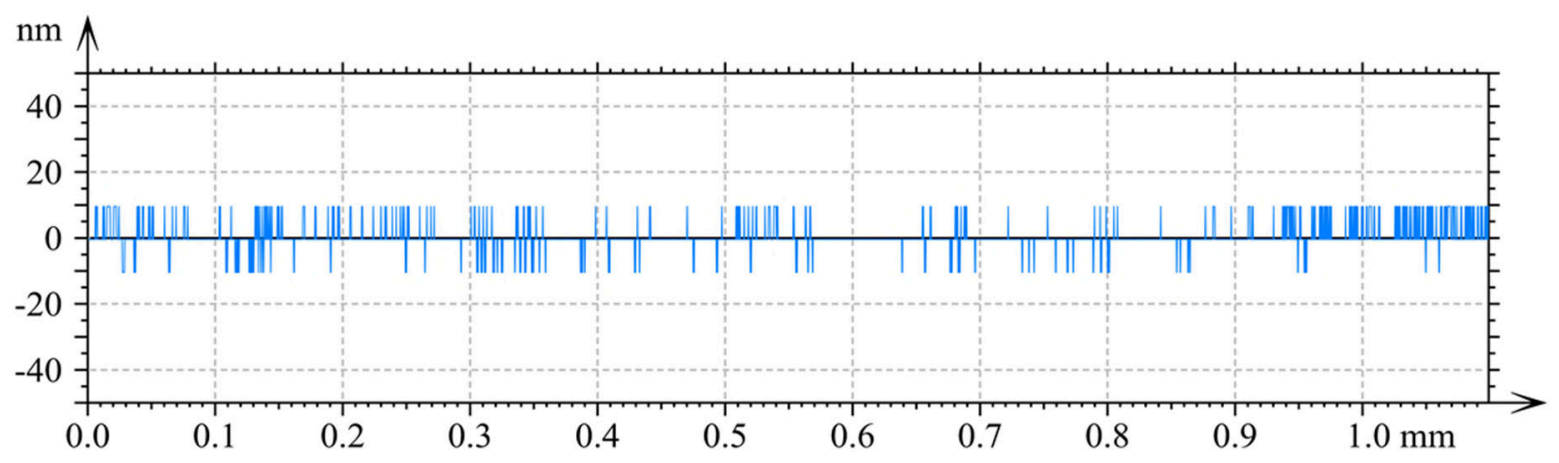

Figure 2 Example of measurement of silicon carbide flat with no tilt (near flat)

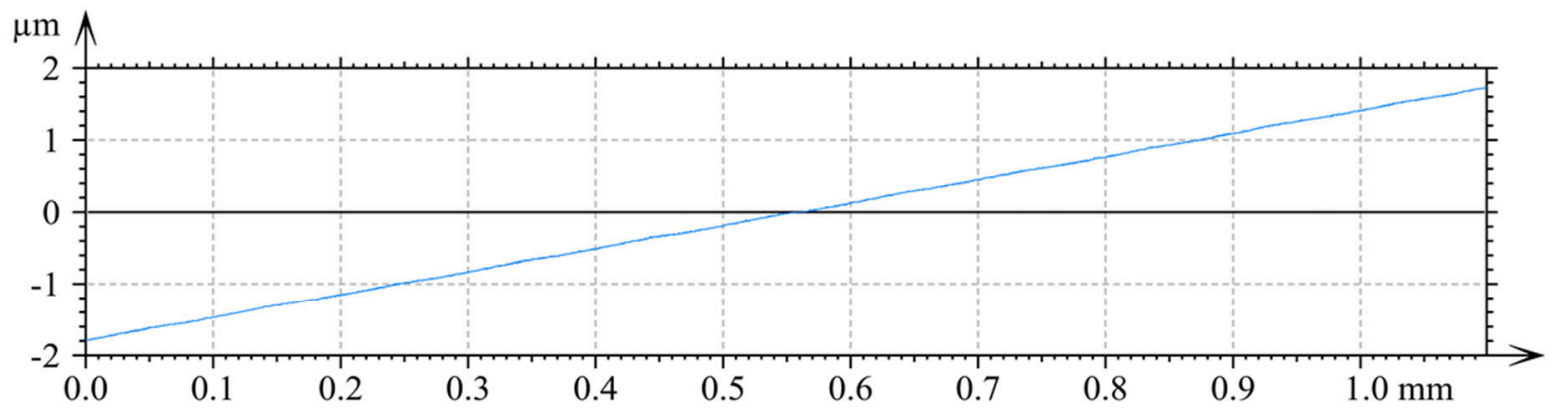

\begin{tabular}{|l|r|l|l|}
\hline Parameters & Value & Unit \\
\hline Length & 1.099 & $\mathrm{~mm}$ & \\
\hline $\mathrm{Pt}$ & 3.520 & $\mu \mathrm{m}$ & \\
\hline
\end{tabular}

Figure 3 Example of measurement of a tilted silicon carbide flat.

The results with a small tilt, estimated at around 12 minutes, that cover around $3.5 \mu \mathrm{m}$ of the $\mathrm{z}$ measurement range are presented in figure 3 (nanometres features are not 
visible due to the tilt). If the measured profile is levelled using a least square line standard operator, and the ordinate values are rescaled using a factor equal to the ratio between the vertical range of $3.52 \mu \mathrm{m}$ and the length of the extracted profile of $1.099 \mathrm{~mm}$, the linearity deviation of the $\mathrm{z}$ scale can be plotted as shown in figure 4, as a function of the $\mathrm{z}$ axis measurement range. The sampling of the linearity in this case is approximately equal to $0.8 \mathrm{~nm}$ - horizontal sampling interval $(250 \mathrm{~nm})$ times vertical range $(3.52 \mu \mathrm{m})$ divided by the length of the extracted profile $(1.099 \mathrm{~mm})$.

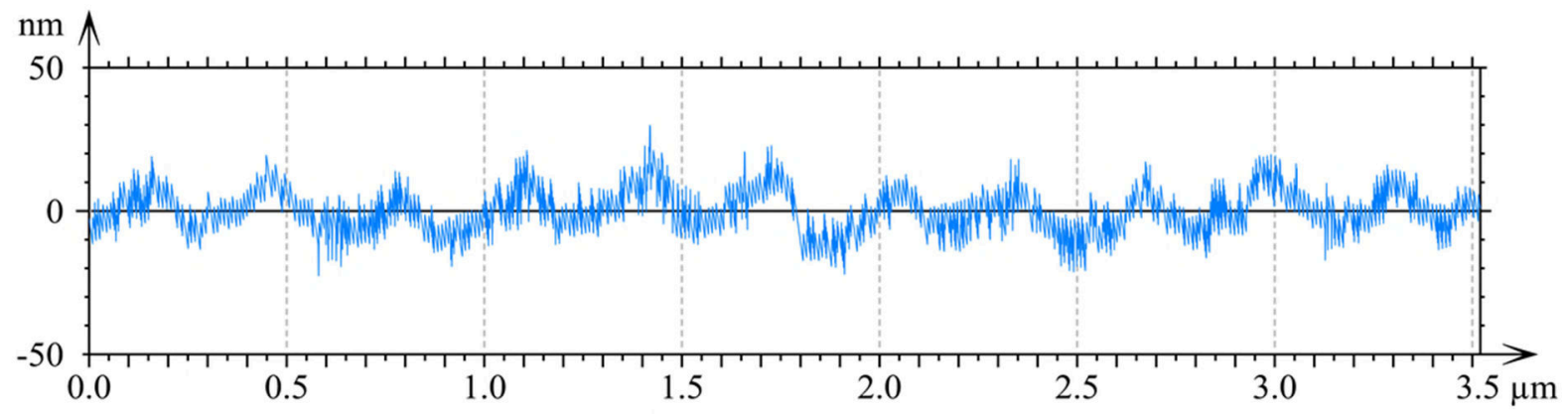

Figure 4 Example of linearity deviation measurement as a function of z measured range obtained from a levelled flat over a $z$ measurement range of $3.5 \mu \mathrm{m}$.

At this stage, it is not obvious from these results whether it corresponds to linearity deviation of the $z$ scale. However, if the errors are related to $x$ scale contribution then the structure seen in figure 4 will only change amplitude for different tilt angles, corresponding to different $\mathrm{z}$ measurement ranges. Conversely, if the errors are arising due to the $z$ scale, then the plot should retain the amplitude but will change laterally. Note that in the later scenario, the pitch will be the same in representation akin to figure 4 , as the following rescaling of the $\mathrm{z}$ measurement range will change.

Figure 5 presents the results of such a test and shows evidence for the above assumptions. The approximate tilt angle of around 26 minutes was measured, which led to an estimation of the linearity deviation over a larger $\mathrm{z}$ range with a nominal sampling interval of $1.9 \mathrm{~nm}$. The main repeating structure remains unchanged in amplitude and in pitch, testifying to the presence of $\mathrm{z}$ scale errors with the exception of a longer wavelength structure, seen as a slight curvature, arising from the $x$ scale error effects.

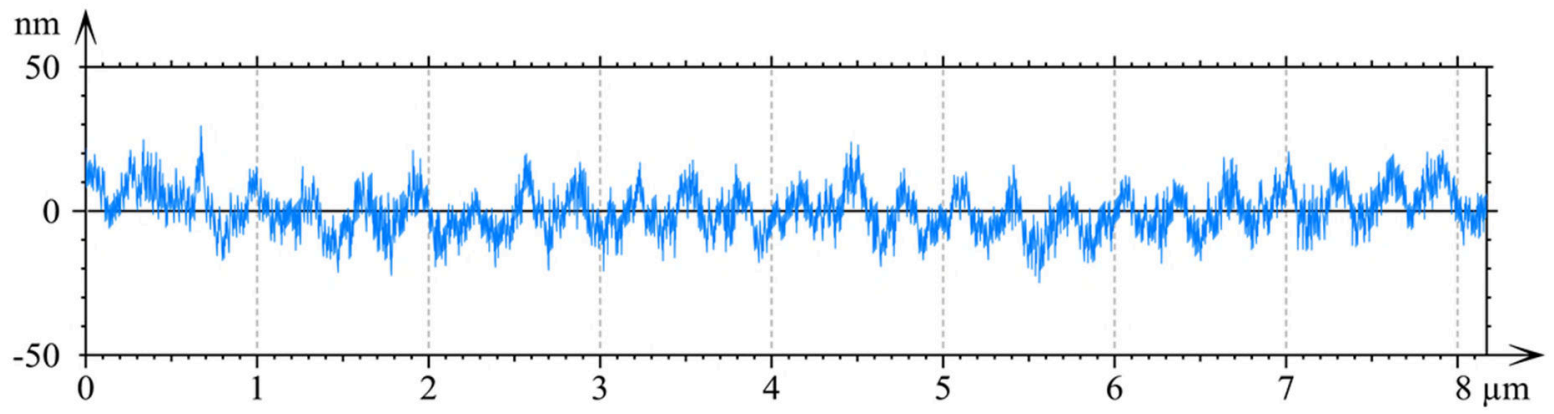

Figure 5 Example of linearity deviation measurement as a function of z measured range obtained from a levelled flat over a $z$ measurement range of $8.1 \mu \mathrm{m}$. 
Nevertheless, the results presented in figure 4 can be used to correct the linearity deviation or estimate the uncertainty associated with the $\mathrm{z}$ measurements performed by the instrument. For example, shifting the profile by a set distance along the $\mathrm{z}$ range and subtracting it from the baseline profile without any shift will give the range of possible measured values corresponding to the shifted distance $\left\{\left(\left(^{\text {ind }-0} \mathrm{Z}_{i+j^{-}} \text {ind }-0 Z_{i}\right)\right\}_{i=1: n-j, j<n}\right.$.

Figure 6 shows the result of such differences when the profile was shifted by $10 \mathrm{~nm}(\mathrm{a})$, $50 \mathrm{~nm}$ (b) and $100 \mathrm{~nm}$ (c). Alternatively, one could plot the histograms of the height distribution. However, the root mean square deviation (RMS) of the profiles provide an estimate of the standard deviation, or variance of the associated PDF if the value is squared.
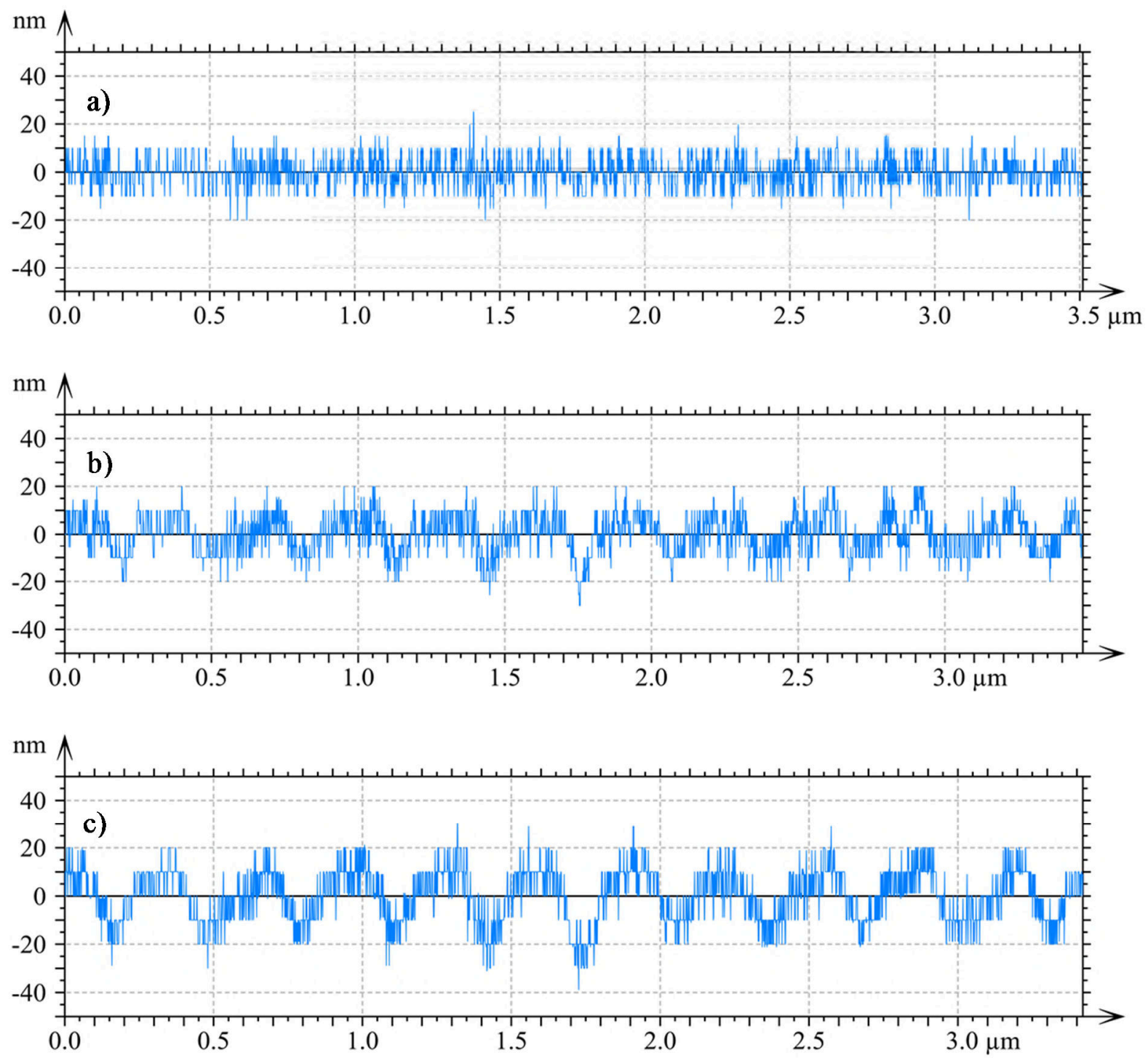

Figure 6 Subtraction results of the profiles shifted with (a) $10 \mathrm{~nm}$, (b) $50 \mathrm{~nm}$ and (c) $100 \mathrm{~nm}$.

The corresponding histograms are presented in figure 7 . The variances for the three cases were estimated to be $29.7 \mathrm{~nm}^{2}, 65.4 \mathrm{~nm}^{2}$ and $112 \mathrm{~nm}^{2}$, respectively. 


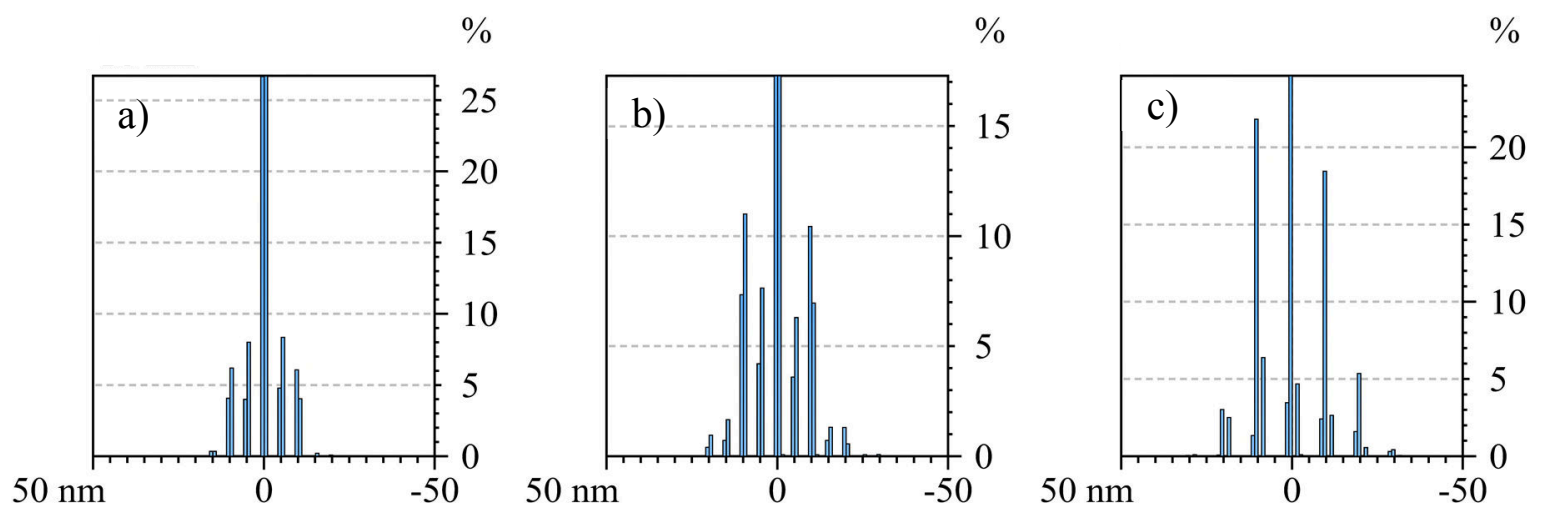

Figure 7 Histograms of potential values associated with the measurement of (a) $10 \mathrm{~nm}$, (b) $50 \mathrm{~nm}$ and (c) $100 \mathrm{~nm}$ lengths along the $\mathrm{z}$ axis. The resolution of the histograms was set to $1 \mathrm{~nm}$.

However, the three histograms include the measurement noise, flatness deviation and sample topography contribution. Similar to the way in which the subtraction results shown in figure 6 were handled, the measurement of a silicon carbide flat with no tilt can be used to estimate the variance associated with the latter three input qualities, and hence to estimate the variances associated with the linearity deviation.

The measurement noise, flatness deviation and sample topography combined variance was found to be $23 \mathrm{~nm}^{2}$, which when subtracted from results obtained from the tilted flat measurements provided the variances for the three cases discussed above, found as $6.7 \mathrm{~nm}^{2}, 42.4 \mathrm{~nm}^{2}$ and $89 \mathrm{~nm}^{2}$, respectively.

To summarise the results, one can conclude that the linearity deviation contribution to the measurement uncertainty associated with $10 \mathrm{~nm}$ height measurement can be propagated in the form of a normal distribution that has an expected value equal to zero and variance equal to $6.7 \mathrm{~nm}^{2}, N\left(0,6.7 \mathrm{~nm}^{2}\right)$.

\section{Discussions}

This method of estimating the linearity deviation of the vertical scale is significantly important, as it enables practitioners to demonstrate with relative ease, the validity and conformity of their results beyond that of traditional scale calibration based on depth measurement standards.

First of all, one basic limitation of the depth standards method is that the traceability of the instrument is limited to the calibrated range determined by the minimum and maximum discrete values reproduced by such standards. The tilted flat method circumvents this issue by mapping the linearity deviations continuously within the range covered by the tilt angle. The full traceability in this case requires the measurement of a single depth measuring standard that can be optimally selected to achieve least uncertainty associated with the amplification coefficient of the vertical scale. For example, figure 4 showed that the linearity of the scale is affected by a sinusoidal error of over $20 \mathrm{~nm}$ in amplitude and $300 \mathrm{~nm}$ pitch. To minimise the effect of the linearity deviation in this case, the calibration of the amplification coefficient should be performed with a depth standard that reproduces in multiples of $300 \mathrm{~nm}$. A step height of about $2.1 \mu \mathrm{m}$ with an associated expanded uncertainty of $10 \mathrm{~nm}$ will allow 
calibrating the vertical scale with an associated expanded measurement uncertainty equal to $11 \mathrm{~nm}$, or $0.5 \%$ of the vertical displacement. Given the cyclic behaviour on the linearity error, we assume here that the linearity at $2.1 \mu \mathrm{m}$ is similar to that calculated for $10 \mathrm{~nm}$ vertical displacement.

To this end it is clear that another advantage of the proposed tilted flat method is that there is no need for multiple depth standards to achieve comprehensive calibration of the $\mathrm{z}$ scale. Even when multiple depth standards are used, the linearity is poorly sampled in comparison with the tilted flat, which is limited by the resolution/digitisation of the $x$ scale times the tangent of tilt angle. For example, AFMs and optical microscopes based on interferometric designs are able to measure atomic steps, however from the point of view of traceability it is difficult to assure standard uncertainties below a nanometre, hindering the use of these instruments to their full potential. The instrument used to provide the results in section 4 is limited by a vertical resolution of $8 \mathrm{~nm}$, which governs the magnitude of the variance associated with linearity contribution. Optical instruments and AFMs are able to measure topographies with noise and resolution in the sub-nanometre range. Hence, an uncertainty contribution of the amplification coefficient equal to $0.5 \%$ of the vertical displacement will have virtually no effect on the measurement of nanometre magnitude features.

The measured profile presented in figure 4 covers a vertical measurement range of $3.5 \mu \mathrm{m}$ sampled at $0.8 \mathrm{~nm}$ intervals. Figure 5 shows that an increase in the tilt angle can extend the calibrated range, however the equivalent vertical sampling distance is also increased to $1.9 \mathrm{~nm}$. A gentler tilt angle is required for finer sampling and a longer profile will provide an increased calibration range. With appropriate design of experiments the linearity of the $z$ scale can be probed with enough resolution that allows derivation of measurement uncertainties fit for purpose.

At the other end of the measurement range, linearity deviation can be probed with ease in an area that sits between the surface texture and micro CMM traceability routes, which is difficult to achieve with small associated uncertainties. Traditionally, extrapolated measurements that are larger than the maximum calibrated value are not allowed. However, the measurement of a tilted flat circumvents this limitation, hence providing sufficient information that allows confidence in measurements performed in the instrument measurement volume rather than limited ranges, and ultimately enabling the full potential use of such instruments. It is noteworthy to mention that not all instruments have the ability to change the sampling and evaluation length or their transfer function is tilt invariant.

\section{Conclusions}

For accurate measurement of surface topography at nanometre level, characterisation of vertical scales of measurement instruments is essential. Current calibration methodologies do not address the demand for sub-nanometre measurement uncertainties at low costs. We propose an approach based on an error separation technique to evaluate accurately the linearity of the vertical scale. The method requires the measurement of an inclined flat sample. We demonstrate that the levelled measured profile provides complete information about linearity deviation and its associated 
probability distribution function, which is of the utmost importance for modern industrial and academic needs. Different tilt angles can provide different sampling and range coverage of the linearity of the vertical scales, not easily achieved with conventional calibration techniques such as the depth standard. With a purposelyselected calibrated depth standard providing traceability to the length unit comprehensive calibration of the vertical scale can be achieved.

We conclude that a simple measured profile can be used to build complex uncertainty budgets without the need of simplified models, which often overestimate or are not fit to serve micro- nanotechnology needs for accurate measurements. For this reason, this method in its simplicity can be considered as a technology enabling tool.

\section{Acknowledgements}

The authors would like to thank Dr Peter Harris (NPL, UK) and Dr Gavin MacAulay (ex NPL) for their helpful discussions and suggestions. This paper also benefited from the fructuous conversations between CLG and members of the Optical Group in ISO TC 213 lead by Dr Theodor Vorburger (NIST, USA). The work was carried out in the Centre for Doctoral Training in Ultra-Precision at Cranfield University which is supported by the RCUK via Grants No.: EP/K503241/1 and EP/L016567/1. SG acknowledge the financial support obtained from other funders such as the RCUK (Grant No. EP/S013652/1), H2020 (EURAMET EMPIR A185 (2018)) and Royal Academy of Engineering (Grant No. IAPP18-19 \295). We also acknowledge financial support from EURAMET EMPIR A185 (2018), which is co-funded by the European Union's Horizon 2020 research and innovation programme and the EMPIR Participating States.

\section{References}

[1] Goel S, Luo X, Agrawal A, Reuben RL. Diamond machining of silicon : A review of advances in molecular dynamics simulation. Int J Mach Tools Manuf 2015;88:131-64.

[2] Uhlmann E, Mullany B, Biermann D, Rajurkar KP, Hausotte T, Brinksmeier E. Process chains for high-precision components with micro-scale features. CIRP Ann - Manuf Technol. 2016;65(2):549-72.

[3 Rosén S, Thomas TR, Rosén B. The Stedman diagram revisited. Surf Topogr: Metrol Prop 2014;2:014005 (5pp).

[4] Cervetti C, Rettori A, Pini MG, Cornia A, Repollés A, Luis F, et al. The classical and quantum dynamics of molecular spins on graphene. Nat Mater. 2016;15:164-8.

[5] Hansen HN, Carneiro K, Haitjema H, Chiffre L De. Dimensional micro and nano metrology. CIRP Ann - Manuf Technol. 2006;55(2):721-43.

[6] Leach RK, Giusca CL, Haitjema H, Evans C, Jiang X. Calibration and verification of areal surface texture measuring instruments. CIRP Ann - Manuf Technol. 2015;64(2):797-813.

[7] Evans CJ, Hocken RJ, Estler WT. Self-calibration : reversal, redundancy, error separation, and "absolute testing". CIRP Ann - Manuf Technol.1996;45(2):617-34. 
[8] Eifler M, Schneider F, Seewig J, Kirsch B, Aurich JC. Manufacturing of new roughness standards for the linearity of the vertical axis - Feasibility study and optimization. Eng Sci Technol an Int J. 2016;19(4):1993-2001.

[9] Cima AH, Morel M, Takawa DH, Phisher H; Mitutoyo Corporation, assignee. Calibration samples of surface texture measuring instrument. Japan patent JP4689988 (B2). 2011-06-01.

[10] Breitmeier U; Testing and / or calibration standard. German patent DE102016122509. 2017-06-01.

[11] Giusca CL, Leach RK. Calibration of the scales of areal surface topography measuring instruments: part 3. Resolution. Meas Sci Technol. 2013;24(10):105010.

[12] MacAulay GD, Giusca CL. Assessment of uncertainty in structured surfaces using metrological characteristics. CIRP Ann - Manuf Technol. 2016;65(1):533-6.

[13] Liu M, Cheung CF, Ren M, Cheng C-H. Estimation of measurement uncertainty caused by surface gradient for a white light interferometer. Appl Opt. 2015;54(29):8670-7.

[14] de Groot P, Beverage J. Calibration of the amplification coefficient in interference microscopy by means of a wavelength standard. In: Bodermann B, Frenner K, Silver RM editors. Modeling Aspects in Optical Metrology V: Proc SPIE. 2015;9526(952610):1-11.

[15] Kiyono S, Gao W, Zhang S, Aramaki T. Self-calibration of a scanning white light interference microscope. Opt Eng. 2000;39(10):2720-5.

[16] ISO12179 BE. BS EN ISO12179. Geometric product specifications (GPS) - surface texture: profile method - calibration of contact (stylus) instruments. British Standards Institute;1996.

[17] Haitjema H, Morel MAA. Accurate roughness measurements by dynamic calibration, VFM-uncertainty calculations and a special calibration specimen. J Phys: Conf Ser. 2005;13:232-5.

[18] Osten W. Optical metrology in the conflict between desire and reality. In: Creath K, Burke J, Albertazzi Gonçalves A, editors. Interferometry XVIII: Proc of SPIE. 2016;9960(99600P):1-8.

[19] JCGM 100:2008. Evaluation of Measurement Data-Guide to the Expression of Uncertainty in Measurement (GUM). Geneva, Switzerland: Joint Committee for Guides in Metrology; 2008.

[20] ISO3274 BE. BS EN ISO3274. Geometric product specifications (GPS) - surface texture: profile method - normal characteristics of contact (stylus) instruments. British Standards Institute;1996. 
2019-09-05

\title{
Improved and simpler estimation of
} scale linearity contribution to topography measurer

\author{
Giusca, Claudiu L.
}

Elsevier

Giusca CL, Goel S. (2019) Improved and simpler estimation of scale linearity contribution to topography measurement. Precision Engineering, Volume 60, November 2019, pp. 368-373 https://doi.org/10.1016/j.precisioneng.2019.09.002

Downloaded from Cranfield Library Services E-Repository 